\title{
GCU
}

Glasgow Caledonian

University

University for the Common Good

\section{MP-SDWN: a novel multipath-supported software defined wireless network architecture}

\author{
Xu, Chuan; Jin, Wenqiang; Han, Yuanbing; Zhao, Guofeng; Tianfield, Huaglory
}

Published in:

Communications and Networking

DOI:

10.1007/978-3-319-66625-9_12

Publication date:

2018

Document Version

Author accepted manuscript

Link to publication in ResearchOnline

Citation for published version (Harvard):

Xu, C, Jin, W, Han, Y, Zhao, G \& Tianfield, H 2018, MP-SDWN: a novel multipath-supported software defined wireless network architecture. in Q Chen, W Meng \& L Zhao (eds), Communications and Networking. Lecture Notes of the Institute for Computer Sciences, Social Informatics and Telecommunications Engineering , vol. 209, Springer, pp. 119-128, 11th EAI International Conference on Communications and Networking in China, Chongqing, China, 24/09/16. https://doi.org/10.1007/978-3-319-66625-9_12

\section{General rights}

Copyright and moral rights for the publications made accessible in the public portal are retained by the authors and/or other copyright owners and it is a condition of accessing publications that users recognise and abide by the legal requirements associated with these rights.

Take down policy

If you believe that this document breaches copyright please view our takedown policy at https://edshare.gcu.ac.uk/id/eprint/5179 for details

of how to contact us. 


\title{
MP-SDWN: A Novel Multipath-Supported Software Defined Wireless Network Architecture
}

\author{
Chuan Xu ${ }^{\mathrm{a}}$, Wenqiang Jin ${ }^{\mathrm{a}}$, Yuanbing Han ${ }^{\mathrm{a}}$, Guofeng Zhao ${ }^{\mathrm{a}}$, Huaglory Tianfield ${ }^{\mathrm{b}}$ \\ ${ }^{\text {a }}$ School of Communication and Information Engineering \\ Chongqing University of Posts and Telecommunications, Chongqing, China \\ ${ }^{\mathrm{b}}$ School of Engineering and Built Environment \\ Glasgow Caledonian University, Glasgow, United Kingdom \\ \{xuchuan, jinwq, hanyb, zhaogf \} @ cqupt. edu. cn \\ h.tianfield@gcu.ac.uk
}

\begin{abstract}
The wireless access networks have witnessed an explosive growth in home and enterprise applications. However, to meet the tough performance requirements, such as seamless handover, higher throughput, and agile programing, there still exists many challenges in WiFi networks. In this paper, we propose a novel MP-SDWN (multipath-supported Software Defined Wireless Network), to provide mobility handover, throughput enhancement, and flow-level transmission control as well as programmable system control interfaces. Furthermore, the effectiveness and efficiency of MP-SDWN is evaluated on an experimental prototype.
\end{abstract}

Keywords: SDN, NFV, MP-SDWN, multipath, handover, flow control

\section{Introduction}

In support of mobility and cloud services provisioning wireless LAN is growing rapidly in quantity as well as its high throughput. The increasing WLAN users bring new requirements, e.g., burst user access, seamless handover, quality of service, high throughput and security. However, most of existing WLAN networks are often deployed in an unplanned and uncoordinated way, and different ISPs often adopt their private protocol and architecture to operate their WLAN systems. As a result, the management and operation of today's WLAN is very inflexible, and with limited support to mobility and specific need of new applications. Meanwhile, WLAN has still suffered from the traditional wireless problems such as radio frequency interference, Ping-Pong effect and energy saving, which are not well solved either.

Software-defined network (SDN) [4-6], an innovative paradigm for programmable network, advocates decoupling the control plane and data plane of networks, which dramatically simplifies network control and enables innovation and evolution by abstracting the control functions of the network into a logically centralized control plane. Moreover, the concept of network function virtualization (NFV) [7-9] effectively separates the abstraction of functionalities from the network hardware and substrate. SDN and NFV technologies provide wireless networks with the needed 
flexibility to evolve according to the ever-changing application contexts. By extending SDN to mobile and wireless networks, it leads to a new trend, i.e. softwaredefined wireless network (SDWN). SDWN is very promising to address challenges in mobile wireless network, such as wireless resource optimization, convergence of heterogeneous networks, fine-grained controllability, and efficient programmability for network innovation and smooth evolution [10].

Recently, SDWN has been studied in wireless access networks and cellular networks with different emphasizes [11-18]. To separate the network service from the underlying physical infrastructure and allow rapid innovation of WLAN services, a number of SDWN architectures are proposed. OpenRoads [11], [12] uses OpenFlow to separate control from the data path through an open API, and FlowVisor to create network slices. OpenRadio [13] proposes a programmable wireless data plane to provide modular programming capability for the entire wireless stack, and utilizes multi-core DSP architecture to achieve NFV functionalities. OpenRF [14] proposes a software defined cross-layer architecture for managing MIMO signal processing with commodity Wi-Fi cards. OpenRF adopts the SDN idea and enables access points to control MIMO signal processing at physical layer. CloudMAC [15] is a distributed architecture, which consists of virtual APs, wireless termination points and an OpenFlow switch. Furthermore, the 802.11 WLAN MAC processing in which is partially performed in data centers on virtual machines connected by an OpenFlow controlled network. Odin $[16,17]$ is an SDN framework that proposes to simplify the implementation of high-level enterprise WLAN services, by introducing light virtual access point (LVAP), which is similar to the virtual access points used in CloudMAC. In [18], the LVAP is extended to a set of programming abstractions to model the fundamental aspects of a wireless network, including state management, resource provisioning, network monitoring, and network reconfiguration. In summary, SDWN architectures mainly focus on abstracting the details of the underlying wireless physical technologies and moving as much as intelligence to the center of the network. However, the additional processing functions required of the less-capable AP devices and centralized controller architecture of SDN might slow down the response to PHY or MAC layer incidents and reduce the WLAN system throughput significantly.

In this paper, to improve the system performance, we propose a novel WLAN system, namely, MP-SDWN, and present the design, implementation, and evaluation. MP-SDWN could provide seamless mobility handover, flow-level QoS control and high throughput for clients as well as programmable interface to meet the needs from enterprise applications and ISP deployments.

The rest of the paper is organized as follows. Section 2 presents the system architecture, and describes typical use cases of MP-SDWN. In Section 3, we evaluate its performance on an experimental prototype. We conclude our work in Section 4. 


\section{MP-SDWN Architecture}

\subsection{Architecture of MP-SDWN}

MP-SDWN is constructed based on SDN and NFV using open-source wireless network devices.

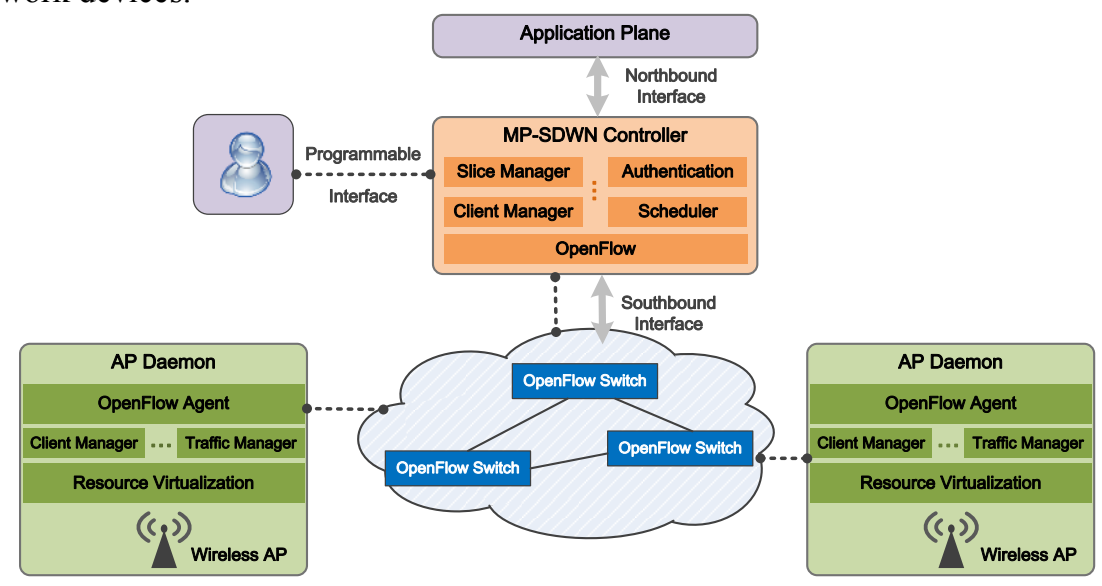

Fig. 1. The architecture of MP-SDWN.

As shown in Figure. 1, MP-SDWN comprises a logically centralized Controller entity and distributed AP Daemons residing on physical AP devices for virtualizing and programming the wireless components, and the Controller and AP Daemons are connected through OpenFlow switch. The entities are described as follows.

Controller. As an SDN controller, the Controller enables applications to orchestrate the underlying physical wireless network entities, which provides a set of interfaces (the northbound interface) to the applications and translates their requests into a set of commands (the southbound interface) to the network devices. It maintains a view of the network components including clients, APs, applications and OpenFlow switches, and provides a set of centralized functions including user authentication; creating, migrating and releasing of MVAP(multi-connection virtual access point, which forms the same virtual AP over different physical APs) for per-client; client and networkslice management; network performance monitoring and resources scheduling; etc.

AP Daemon. AP Daemon runs on the physical APs, executes the command from the Controller to orchestrate the wireless network, measures and reports the MVAP performance of Clients on APs. Firstly, it takes over the WiFi devices and virtualizes high-level wireless functions of 802.11 MAC protocol for different network slices; secondly, it maintains MVAP and WFTR for each client, dispatching and forwarding his/her traffic flow according to flow-tables in MVAP, and differentiating flow priorities among diverse applications according to the rules in WFTR; thirdly, as the status of client changes frequently, it sniffers the wireless frames in real time for 
monitoring the performance of MVAPs, to support a publish-subscribe information system when a certain frame event triggered.

Programmable Interface. We provide a set of open programmable interfaces for systems administrator, they can create multiple independent network slices, and inject their own routing or scheduling algorithms into network slices. Moreover, applications can access the statistics at different granularity including frame, flow, MVAP, client, OpenFlow and network slice. The programmable interface allows administrator to share the network control, e.g., load balancing, troubleshooting, and supports researchers to inject customized routing, flow assignment or migration algorithm into MP-SDWN core module to testify their idea. In addition, the performance of customized algorithm can be observed through the performance monitoring module.

In summary, the major design considerations of the MP-SDWN are as follows.

- Firstly, a unified and extended SDN and NFV abstraction, multi-connection virtual access point (MVAP) is introduced to simplify the complexity of IEEE 802.11 protocol stack and to move the operation of protocol stack into application layer, and facilitate easy handling and migration of per-client state cross different physical APs. Different from the single VAP abstraction, MVAP maintains the same VAP for each client on several adjacent physical APs simultaneously, to support multiple connections from several physical APs when client migrates or stays in the wireless signal overlap area. Furthermore, MVAP adds a collaborative hierarchical flow table distributed on all VAPs for each client, to forward all flows from the client in parallel to improve client's throughput.

- Secondly, we design and implement a wireless flow transmission rule table (WFTR) into mac80211 subsystem to achieve wireless transmission control in flowlevel, similar to the packet forwarding control on wired network, which allows administrators to differentiate flow priorities of diverse applications from the same user. Adding control parameters including transmission power, transmission rate and priorities into WFTR, MP-SDWN can provide flow-level QoS control to satisfy the needs from various applications.

- Thirdly, MP-SDWN introduces a programmable interface which allows ISPs to share the network control, e.g., load balancing, QoS policy, troubleshooting. Moreover, the interface supports researcher to inject customized routing or scheduling algorithm into MP-SDWN core module to simulate their idea. We implement MPSDWN, and evaluate the feasibility and efficiency of our framework by conducting several typical WiFi application experiments using the prototype deployed at our university.

\subsection{Use Cases}

MP-SDWN is designed to provide seamless access in the dense deployment scenarios, and there typical use cases are shown in Fig. 2. 


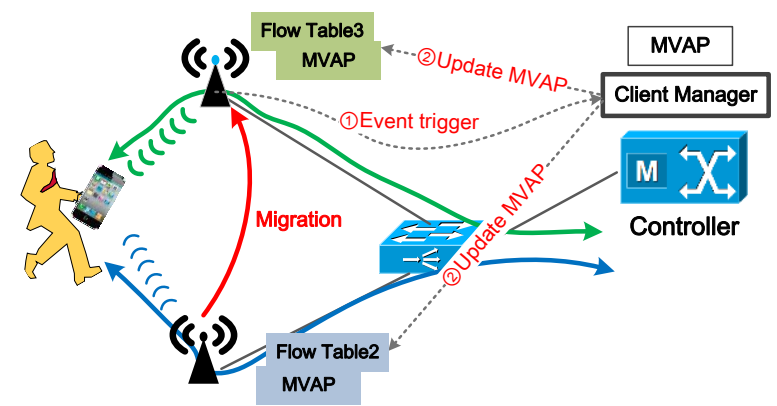

(a) Mobility and migration, the state and MVAP of client can be migrated seamlessly or work on the two APs simultaneously during the handover.

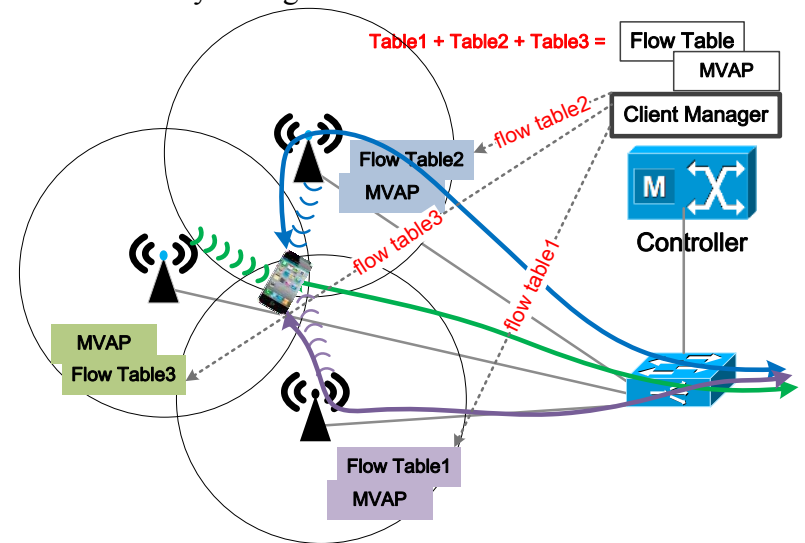

(b) Multipath transmission, for each client, multiple connections and transmission paths are provided when it stays in the wireless signal overlap area of several APs.

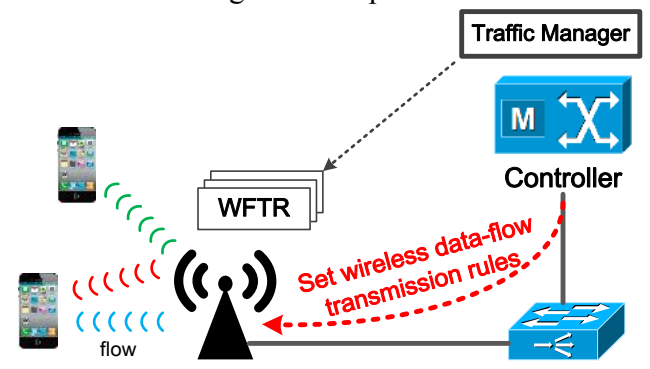

(c) Transmission control, administrator can set specific wireless transmission rules for perflow to each client through the controller.

Fig. 2. The typical use cases based on MP-SDWN.

Mobility and Migration. Based on the virtualization of 802.11 MAC protocol, MPSDWN supports seamless user mobility and dynamic resource management for each client. During the handover, the changes in wireless signal strength are captured on nearby APs, then the migration event will be triggered and send to Controller by APs, 
and at last, the Controller will dispatch the resources according to the status of mobility and performance demand from the client.

Multipath Transmission. When the client roams around the wireless signal overlap area from several adjacent physical APs, MP-SDWN provides a unified abstraction MVAP to support multiple connections from these adjacent physical APs for the client. Based on the MVAP mechanism, all the traffic from the client can be assigned and transmitted simultaneously on multiple paths in flow-level, which can improve the throughput obviously.

Transmission Control. MP-SDWN supports flow-level wireless transmission control for different applications from same user. Through the programmable interface, network administrator can use the WFTR table to set the wireless transmission parameters, such as transmission rate, power, retransmission and RTC/CTS strategy, to per-packet.

\section{Performance Evaluation}

\subsection{Test-bed Setup}

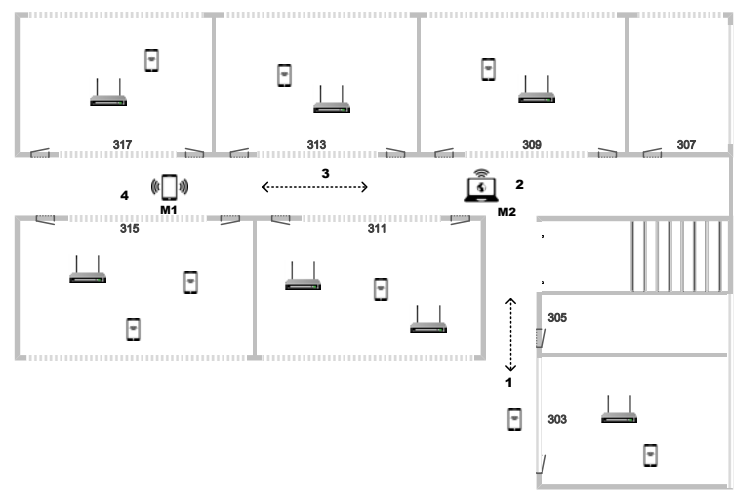

Fig. 3. The deployment of MP-SDWN system in YiFu building.

MP-SDWN has been implemented and deployed in the real network at third floor of YiFu building in CQUPT campus. As shown in Figure 3, the deployment consists of 20 IEEE 802.11n enabled APs, distributed in each office room across this floor, connected with the OpenFlow switchers to the Controller. Furthermore, all APs are restricted to the same channel on $2.4 \mathrm{GHz}$ band. We also deployed two test mobile devices and a number of random mobile devices, one is an android mobile phone (M2), and another is a notebook (M1). We set four observation points to collect the data from controller when the test devices move about. 
Deployed on Netgear WNDR3800 device, APs run OpenWRT release 14.07 with the ath9k Linux driver, the embeded linux version is 3.10.49, user-level click modular router 2.0.1 [19], and OpenvSwitch (OvS) version 2.3.90 supporting OpenFlow (OF) version 1.3. MP-SDWN controller runs on Ubuntukylin-14.04 with floodlight version 1.2 with dual 4-cores CPU and 16G RAM Dell Server supported.

\subsection{Benchmark Performance of MVAP}

As a key technology to provide mobility and performance for users in MP-SDWN, we perform experiments to measure the effectiveness of MVAP. The goal is to understand the benchmark performance for mobile user, including user association and performance improvement.

\section{Delay of Association}

For each client, association is the first step to connect to the WLAN network. Different to traditional WLAN, MP-SDWN needs to create new MVAP identification and choose APs to serve for the client, and the performance of association will affect the user experience directly. Regarding, we compare the latency to standard WiFi system without authentication.

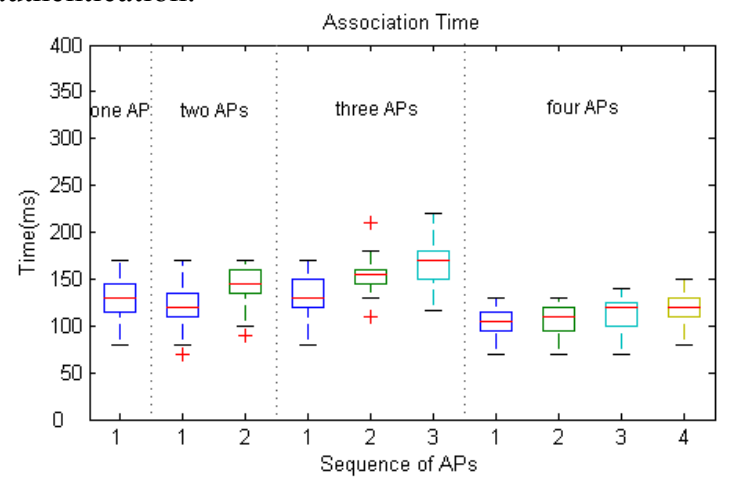

Fig. 4. The Delay of association.

In MP-SDWN system, we provide several APs' connection to the client according to the position where the client stays. To the client, the association can be established when the first connection finishes, and the client only perceives one connection although other connections running in the background. Figure 4 shows the time by which the association is established successfully to the client with different number of connections to APs. For regular WiFi, the association time is around $100 \mathrm{~ms}$. In our system, for one AP, most of the association time is slightly larger than $100 \mathrm{~ms}$, the median value is $125 \mathrm{~ms}$; for two APs, the association time is smaller than the value with one AP, and the median value is $118 \mathrm{~ms}$, and the second AP establishes the connection which is $20 \mathrm{~ms}$ after the first one; but for four APs, to our surprise, the association time is very stable and close to the regular $\mathrm{WiFi}$, the median value is $102 \mathrm{~ms}$, and the second, third and fourth APs are connected to the client within $10 \mathrm{~ms}$. 
Evidently, MVAP sets up more connections serving the client, and the total association time is almost the same as the regular WiFi.

Performance Improvement with MVAP

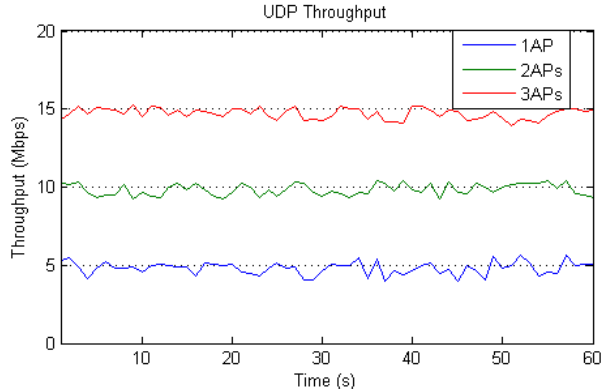

(a) UDP throughput with several APs.

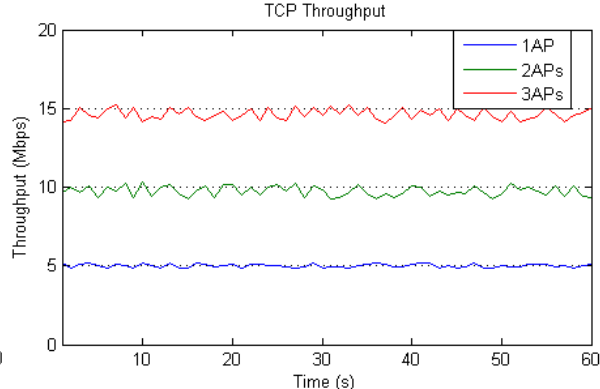

(b) TCP throughput with several APs.

Fig. 5. Performance improvement with MVAP.

Since the MVAP provides multiple paths to transmit the traffic from the client, it will improve the performance significantly especially when the bandwidth from a single AP cannot satisfy the throughput requirement of client. To verify the performance improvement, we restrict the bandwidth of each AP to $5 \mathrm{Mbps}$, and use Iperf on the client to generate several different UDP and TCP flows. When slowly moving the client from observation point 1 to 4 , we obtain the throughput changes from Iperf.

The UDP throughput is shown in Figure 5 (a), when only one AP serving the client, due to the bandwidth constraint, the throughput varies around 5Mbps. Afterwards, when more APs serving the client, the throughput increases to 10Mbps with two APs and nearly $15 \mathrm{Mbps}$ with three APs. As shown in Figure 5 (b), similar to UDP throughput, the TCP throughput increases with more APs serving the client, 10Mbps with two APs and nearly $15 \mathrm{Mbps}$ with three APs. Since the wireless environment is unstable when the client moves about, the throughput increases with fluctuations, nevertheless, the TCP throughput is more stable than the UDP throughput.

\subsection{Handover Performance}

To assess the effectiveness of handover performance, we consider a standard WiFi network as the baseline scenario. So we switch the MP-SDWN and the standard WiFi system running on the APs, and choose observation points 1 and 2 to perform the evaluation, where the handover will be triggered when the client is moving a round trip between the two. In a standard WiFi network, handover is triggered by the wireless clients when the RSSI decreases and the network cannot control the mobility of wireless clients. 


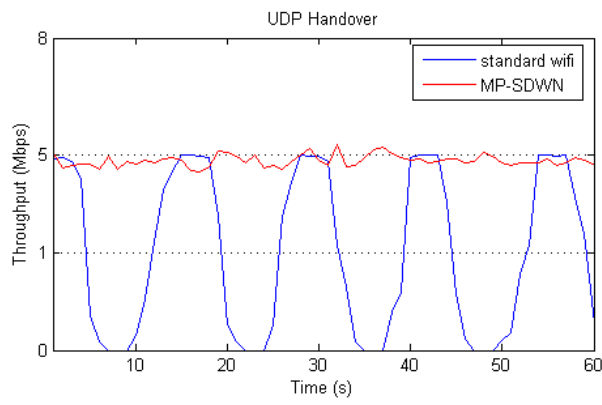

(a) UDP throughput.

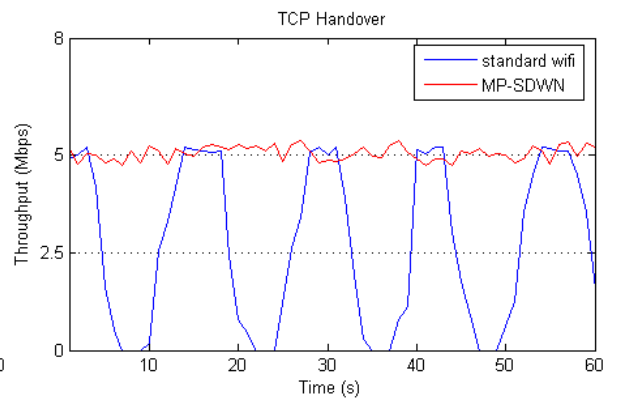

(b) TCP throughput.

Fig. 6. Throughput at the receiver side when the client is performing a round trip handover.

The traffic is generated at the wireless client through Iperf with a fixed speed $5 \mathrm{Mbps}$. Figure 6 shows the distribution of throughput at the receiver's side when the client is performing a round trip handover. As shown in Figure 6 (a), the UDP throughput of standard wifi performs a significant degradation periodically. As the handover is triggered, the client would take 1-2 seconds to re-establish the association with the new AP, which can effectively lead to the throughput degradation. We also tested the handover performance with TCP traffic, the same results are observed in Figure 6 (b). On the other hand, with completely seamless handovers supported by the MP-SDWN system, the UDP and TCP throughputs remain stable on 5Mbps.

\section{Conclusion}

To improve the performance of WLAN networks, we have proposed a novel WLAN system based on SDN and NFV, called Multipath-Supported Software Defined Wireless Network (MP-SDWN) to meet the needs from different applications. First, the MVAP simplifies the complexity of IEEE 802.11 stack and handle per-client state cross different physical APs. Take the advantage of the multiple connections on several adjacent APs simultaneously, client can obtain high throughput whether it migrates or stays in the wireless signal overlap area. Then, the WFTR running in mac80211 subsystem provides wireless transmission control in flow-level to meet the QoS requirements from different applications on the same client. Lastly, the programmable interface is presented for network administrator to exert the network control, e.g., load balancing, QoS policy, troubleshooting. We have deployed the system in an experimental network to evaluate the performance improvement and stability of MP-SDWN, and believed that the home and enterprise networks can benefit from the capabilities as demonstrated in this research.

Acknowledgments. This work is supported by the National Science Foundation of China (NSFC) under grant 61402065, Chongqing Key Science and Technology Program (No.cstc2014jcsf70001), the China Scholarship Council and Young Backbone Academics Funding Program of Chongqing Municipal Education Commission. 


\section{References}

1. Cisco. Cisco Service Provider Wi-Fi.: A Platform for Business Innovation and Revenue Generation. J. In Cisco, 2015.

2. P. Valerio.: Using carrier wifi to offload iot networks. J. In InformationWeek: Network Computing, 2014.

3. CNNIC.: $37^{\text {th }}$ Development Statistics Report of Internet Network in China. U. https://www.cnnic.net.cn, 2016

4. Yu M, Rexford J, Freedman MJ, Wang J.: Scalable flow- based networking with difane. J. SIGCOMM Comput Commun Rev 41(4):351-362, 2015

5. Kim H, Feamster N.: Improving network management with software defined networking. J. IEEE Commun Mag 51(2):114-119, 2013

6. McKeown N, Anderson T, Balakrishnan H, Parulkar G, Peterson L, Rexford J, Shenker S, Turner.: Openflow: enabling innovation in campus networks. J. SIGCOMM Comput Commun Rev 38(2):69-74, 2008

7. Chowdhury N, Boutaba R.: Network virtualization: state of the art and research challenges. J. IEEE Commun Mag 47(7):20-26, 2009

8. Yang M, Li Y, Jin D, et al.: Software-Defined and virtualized future mobile and wireless networks: a survey. J. Mobile Networks and Applications, 20(1): 4-18, 2014

9. Chowdhury NMK, Boutaba R.: A survey of network virtualization. J. Comput Netw 54(5):862-876, 2012

10. Astuto BN, Mendon M, Nguyen XN, Obraczka K, Turletti T.: A survey of software-defined networking: past, present, and future of programmable networks. J. IEEE Commun Surv Tutor 16(3):1617-1634, 2014

11.Yap KK, Kobayashi M, Sherwood R, Huang TY, Chan M, Handigol, N, McKeown N.: Openroads: empowering research in mobile networks. J. SIGCOMM Comput Commun Rev 40(1):125-126, 2010

12. Yap KK, Sherwood R, Kobayashi M, Huang TY, Chan M, Hand- igol N, McKeown N, Parulkar G.: Blueprint for introducing innovation into wireless mobile networks. C. In: Proceedings of the 2nd ACM SIGCOMM workshop on virtualized infrastructure sys- tems and architectures, ser. VISA '10. USA, New York, pp.25-32, 2010

13. Bansal M, Mehlman J, Katti S, Levis P.: Openradio: a programmable wireless dataplane. C. In: Proceedings of the 1st work- shop on Hot topics in software defined networks, ser. HotSDN '12, New York, pp 109-114, 2012

14. Kumar S, Cifuentes D, Gollakota S, Katabi D.: Bringing cross-layer mimo to today's wireless lans. J. SIGCOMM Comput Commun Rev 43(4):387-398, 2013

15. Dely P, Vestin J, Kassler A, et al.: Cloudmac - an Openflow based architecture for 802.11 MAC layer processing in the cloud. C. GlobeCom Workshops, 2012 IEEE. IEEE: 186-191, 2012

16. Suresh L, Schulz-Zander J, Merz R, Feldmann A Vazao T.: Towards programmable enterprise wlans with odin. C. In: Proceed- ings of the 1st workshop on hot topics in software defined networks, ser. HotSDN '12, New York, pp.115-120, 2012

17. Schulz-Zander, Julius, et al.: OpenSDWN: Programmatic control over home and enterprise WiFi. C. Proceedings of the 1st ACM SIGCOMM Symposium on Software Defined Networking Research. ACM, pp.1-12, 2015

18. Riggio R, Marina M K, Schulz-Zander J, et al.: Programming abstractions for softwaredefined wireless networks. J. Network and Service Management, IEEE Transactions on, 12(2): 146-162, 2015

19. Click modular router project. http://read.cs.ucla.edu/click. 